\title{
Management of a cervical spine neurenteric cyst via an anterior surgical approach: illustrative case
}

\author{
Arsalaan Salehani, MD, Sasha Howell, MD, and Daniel Harmon, MD \\ Department of Neurosurgery, University of Alabama at Birmingham Hospital, Birmingham, Alabama
}

\begin{abstract}
BACKGROUND Central nervous system neurenteric cysts (NCs) represent a rare entity thought to arise from failure of the separation of endodermal and neuroectodermal elements during week 3 of embryogenesis. They account for $0.7-1.3 \%$ of all spinal cord lesions and are typically intradural extramedullary lesions located near the cervicothoracic junction. Most NCs are associated with multisystem malformation disorders, making a solitary extramedullary NC a rare entity.

OBSERVATIONS A 45-year-old man presented with progressive right lower-extremity weakness and an inability to walk. Cervical spine magnetic resonance imaging demonstrated an approximately $1.6 \times 1.1 \times 2.7-\mathrm{cm}$, T2 hyperintense, nonenhancing, intradural, extramedullary cystic lesion at the level of C6-7 eccentric to the right with atrophy of the spinal cord. An anterior surgical approach was used for resection of the cyst in totality with C6-7 corpectomies and anterior plating and fixation from C5 to T1. Postoperatively at 1 month, the patient denied any significant neck or arm pain and demonstrated improving right lower-extremity strength, allowing some funcitonal independence.
\end{abstract}

LESSONS A solitary, extramedullary cervical NC is a rare entity, with a posterior surgical approach for resection primarily described in the literature. The authors present anterior corpectomy and plating with fixation as a viable surgical approach for this rare pathology.

https://thejns.org/doi/abs/10.3171/CASE20119

KEYWORDS cervical; spine; neurenteric cyst

Central nervous system neurenteric cysts (NCs) represent a rare entity thought to arise from failure of the separation of endodermal and neuroectodermal elements during week 3 of embryogenesis, resulting in thin-walled cysts with a lining similar to the respiratory or gastrointestinal epithelium. ${ }^{1,2}$ They account for $0.7-1.3 \%$ of all spinal cord lesions and are typically ventral in location. $^{3}$ The vast majority of cysts are intradural, extramedullary and are typically associated with multisystem malformation disorders, including vertebral and cutaneous anomalies. ${ }^{4,5}$ They are typically located in the lower cervical and upper thoracic spine and demonstrate hyperintensity on T2-weighted magnetic resonance imaging (MRI) sequences. ${ }^{6,7}$ Solitary spinal NCs are rare entities, with the published literature indicating older age and cervical location to be correlated with their presence, whereas younger age and lumbosacral location are correlated with cases associated with dysraphism. ${ }^{2}$ We present a rare case of a middle-aged man with solitary cervical $\mathrm{NC}$ without associated dysraphism or malformation disorder.

\section{Illustrative Case}

The patient was a 45-year-old man with a history of hypertension and obesity (body mass index $33 \mathrm{~kg} / \mathrm{m}^{2}$ ). He was referred to the neurosurgery clinic for evaluation of right lower-extremity weakness and an inability to walk. He reported a constant aching, throbbing, severe neck and bilateral upper-extremity pain that had begun several months before presentation. He described symptom resolution initially with muscle relaxers; however, approximately 1 month before presentation, he awoke suddenly with an inability to bear weight on his right leg. In addition, he developed numbness and tingling from the waist down to his feet with progression to being constantly present and worse on the left side. Given the progression of these symptoms and the persistence of his right leg weakness, he became a wheelchair user, for which he was referred to a neurologist. Subsequent MRI demonstrated a cervical spine cystic lesion, which prompted referral to neurosurgery for tertiary care.

At the time of initial evaluation, the patient denied any neck or arm pain but was experiencing incontinence due to an inability to ambulate

ABBREVIATIONS MRI = magnetic resonance imaging; $N C=$ neurenteric cyst; $P O D=$ postoperative day INCLUDE WHEN CITING Published February 1, 2021; DOI: 10.3171/CASE20119.

SUBMITTED November 15, 2020. ACCEPTED December 1, 2020.

(C) 2021 The authors, CC BY-NC-ND 4.0 (http://creativecommons.org/licenses/by-nc-nd/4.0/) 


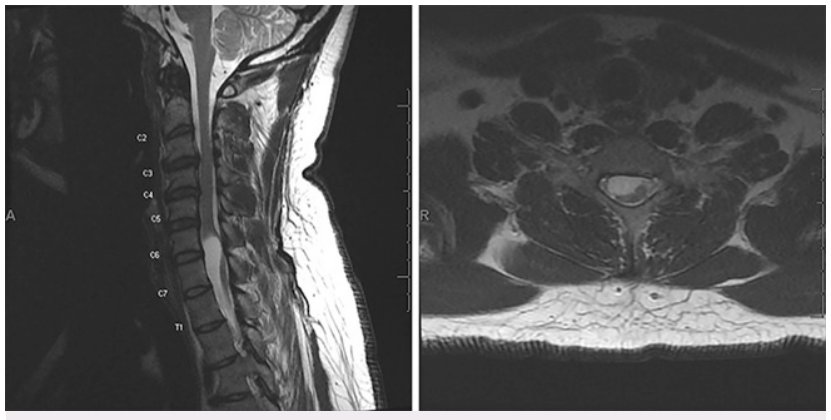

FIG. 1. T2 sagittal (left) and axial (right) cervical MRI. T2 hyperintense intradural, extramedullary cystic lesion at the level of C6-7 eccentric to the right with atrophy of the spinal cord.

to the bathroom in time, forcing the need for a urinal. His neurological examination was significant for left extensor hallucis longus and left foot dorsiflexion $4 / 5$ strength and right lower extremity 4-/5 strength throughout. In addition, he was experiencing decreased sensation to light touch in his left lower extremity but had intact sensation in his right lower extremity. He was also hyperreflexic in both legs and refused to ambulate because of gait instability. MRI was repeated, with additional contrast imaging demonstrating an approximately $1.6 \times 1.1 \times 2.7-\mathrm{cm} \mathrm{T2}$ hyperintense intradural, extramedullary cystic lesion at the level of $\mathrm{C} 6-7$ eccentric to the right with atrophy of the adjacent spinal cord (Fig. 1). Neither the lesion nor the surrounding spinal cord demonstrated contrast enhancement (Fig. 2).

It was recommended that the patient undergo resection, given persistent and progressive neurological compromise due to this suspected NC. The risks and benefits of surgery were discussed with the patient and his family, and he elected to proceed. Given the primarily ventral location of the cyst and the sliver of spinal cord situated posterior to the inferior portion of the cyst (Fig. 1), an anterior approach was deemed safer with an anticipated higher likelihood of complete cyst resection. Discectomies were completed at C5-6, C6-7, and $\mathrm{C} 7$ to T1. A rongeur was used to begin the corpectomies at $\mathrm{C} 6$ and $\mathrm{C} 7$, which were then completed with a high-speed drill. The posterior longitudinal ligament was then removed with a curette and Kerrison punch. The dura was then opened with a number 11 blade, followed by opening of a fairly scarred arachnoid once hemostasis
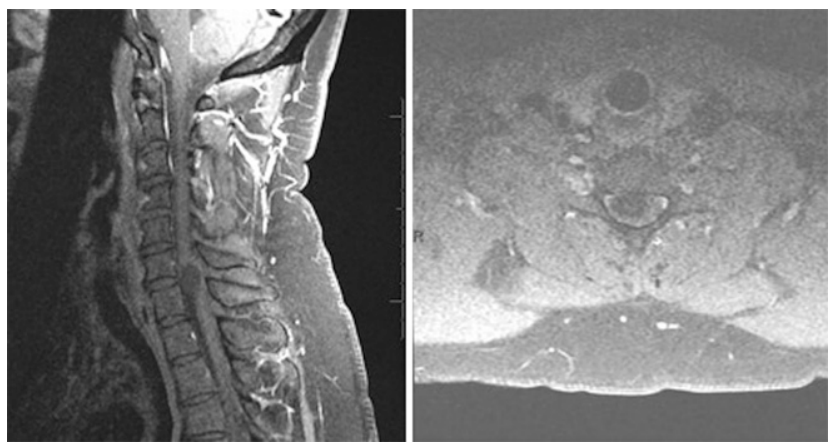

FIG. 2. T1-contrasted sagittal (left) and axial (right) cervical MRI. Nonenhancing, intradural, extramedullary cystic lesion without enhancement of surrounding spinal cord.

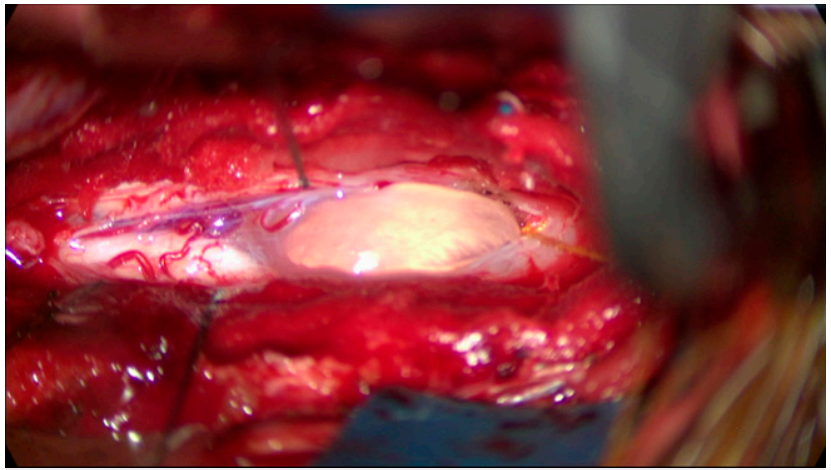

FIG. 3. Intraoperative microscopic view of a large, intradural extramedullary cyst deforming the spinal cord to the right.

was achieved. A large cyst was found underneath this plane, deforming the spinal cord (Fig. 3). A dissection plane was found inferior to the cyst along with a vascular pedicle entering the cyst and connecting to a second artery overlying the cyst. The inferior portion of the cyst was morselized and sent for frozen pathology, which determined that it was an NC. With the use of microsurgical resection techniques, the cyst wall was carefully dissected from the spinal cord, which appeared to be splayed and compressed by the cyst. The vascular pedicle entering the cyst superiorly and inferiorly was isolated, coagulated, and divided, allowing complete resection of the cyst (Fig. 4). The dura was then closed, followed by placement of an expandable cage filled with bone graft and bone harvested from the corpectomies. Finally, an anterior plate spanning from C5 to T1 was then placed and secured with screws.

The patient was admitted to the neurological intensive care unit postoperatively for mean arterial pressure management with a goal of 80-95 mm Hg. On postoperative day (POD) 1, he reported right shoulder pain, for which radiography was performed but did not demonstrate any abnormalities other than degenerative changes. The surgical Hemovac drain was removed on POD 2. Neurontin and lidocaine patches were added to the patient's pain regimen, with some improvement in right shoulder pain achieved before discharge. Compared with his weakness preoperatively, his postresection motor strength in the bilateral lower extremities was $5 / 5$. The patient worked

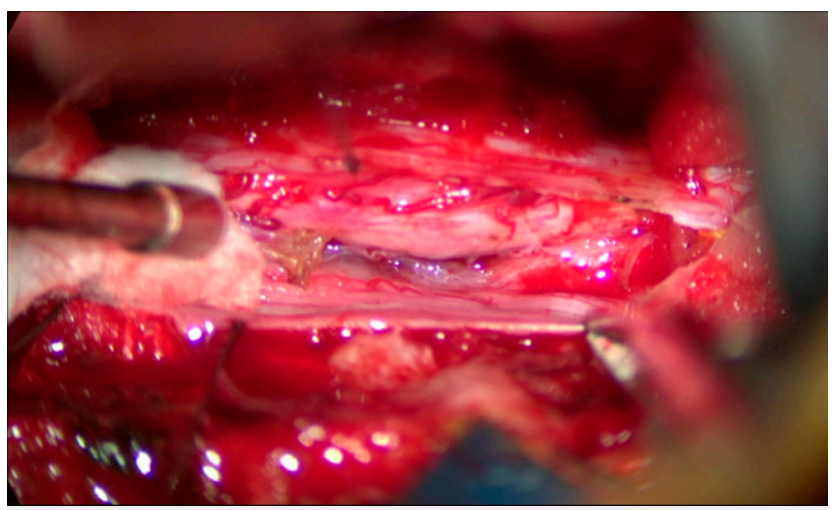

FIG. 4. Intraoperative microscopic view after resection of an intradural extramedullary cyst with demonstrated spinal cord deformation. 

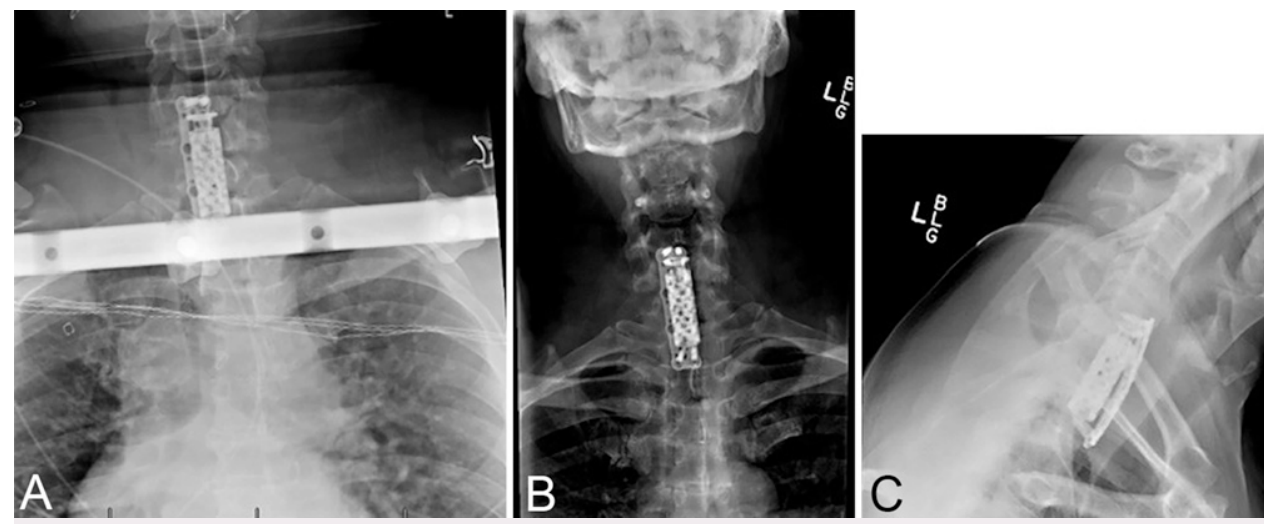

FIG. 5. Intraoperative (A) and 1-month postoperative cervical spine anteroposterior (B) and lateral (C) radiographs demonstrating appropriate spinal alignment and stable hardware position.

with physical and occupational therapists, who recommended inpatient rehabilitation after discharge because of continued gait instability. The patient and his family requested referral to a rehabilitation facility closer to home, so he was discharged on POD 7 to an inpatient rehabilitation facility near his home in Pensacola, Florida. The final pathology was NC.

The patient then returned for a 1-month postoperative checkup and was doing well overall without any significant neck or arm pain. He did have persistent right shoulder blade discomfort that limited his ability to raise his right arm completely over his head; however, this has improved since surgery. He successfully completed his inpatient rehabilitation stay near Pensacola and is now working with home health physical therapy. His speech and swallowing are functioning normally, and he feels that his right lower-extremity strength has improved since before surgery, allowing him to ambulate some on his own. Cervical spine radiography performed at follow-up demonstrated appropriate spinal alignment and hardware position without concern for loosening or failure (Fig. 5). He will return to the clinic periodically for follow-up and surveillance MRI.

\section{Discussion \\ Observations}

Isolated extramedullary NC without congenital syndromic origin remains a rare presentation of acute myelopathy in adults. These tumors are 1.5-3 times more common in males than in females and are typically found in infancy or in the second to third decades of life. ${ }^{4}$ During the third week of embryogenesis, the failure of separation between the endoderm and neural ectoderm results in an intradural growth of endodermal cells within the neuroaxis. NCs are typically lined by nonciliated, simple, pseudostratified, and cuboidal or columnar epithelium with cells characteristically secreting large amounts of proteinaceous material. ${ }^{1,2}$ The T2 hyperintense signaling on MRI scans is thought to be a reflection of these histopathological features. ${ }^{8}$

NCs are most commonly ventrally located within the neural axis. However, despite this anatomical predilection, lateral, posterior, and anterior surgical approaches have been reported with variable success in complete resection. ${ }^{9}$ Factors to consider before surgical planning include identification of intramedullary versus extramedullary growth in addition to location within the spinal canal. Specifically, extramedullary lesions frequently have identifiable dissection planes, allowing a higher rate of total resection. In contrast, intramedullary dissection planes can be more difficult to identify. ${ }^{10}$ Posterior approaches using cervical and thoracic laminectomies followed by fusion, if indicated, remain the most commonly described management of these lesions. ${ }^{9}$

In the current case, however, an anterior approach was selected with resultant 66-7 corpectomy for resection of an extramedullary mass with $\mathrm{C} 5$ through $\mathrm{T} 1$ anterior fixation and fusion with plating. This anterior approach was believed to provide the greatest opportunity for complete resection, given the eccentricity of the cyst to the right causing spinal cord atrophy. To our knowledge, this particular surgical approach for $\mathrm{NC}$ resection has not been described in recent literature. A similar approach was described by Kida and colleagues in 2018 for a C3-4 NC treated with subtotal $\mathrm{C} 3$ and $\mathrm{C} 4$ corpectomies, but anterior cervical fusion after tumor resection was not attempted. ${ }^{11}$

Although the choice of surgical approach remains controversial in the literature, the ultimate goal of complete resection is largely agreed upon. Rates of recurrence for $\mathrm{NCs}$, regardless of location, ranged from $0 \%$ to $37 \%$ in a recent case series and literature review. ${ }^{6}$ Some studies suggest higher rates of recurrence with a lack of cyst wall resection. ${ }^{12}$ Therefore, in our case, the complete cyst wall was resected. Our patient was doing well at the first follow-up and has been scheduled for periodic follow-up with MRI to monitor for recurrence.

\section{Lessons}

We present the first case of complete cervical corpectomy and anterior fixation with plating for resection of a ventral lower cervical spinal NC. The eccentric location of the cyst with associated spinal cord atrophy made this anterior approach more favorable than the more common posterior approach for this pathology. We hope our contribution to the literature provides our colleagues with an additional technique in their surgical armamentarium.

\section{References}

1. Kimura $\mathrm{H}$, Nagatomi $\mathrm{A}$, Ochi M, et al. Intracranial neurenteric cyst with recurrence and extensive craniospinal dissemination. Acta Neurochir (Wien). 2006;148(3):347-352.

2. Paleologos TS, Thom M, Thomas DG. Spinal neurenteric cysts without associated malformations. Are they the same as those presenting in spinal dysraphism? Br J Neurosurg. 2000;14(3):185-194.

3. El Ahmadieh TY, Sillero R, Kafka B, et al. Isolated dorsal thoracic neuroenteric cyst with spinal cord compression: case reports in pediatrics. World Neurosurg. 2018;118:296-300. 
4. Bruzek AK, Kucia EJ, Oppenlander ME. Intramedullary and extramedullary cervical neurenteric cyst requiring fixation and fusion. World Neurosurg. 2016;95:621.e7-621.e12.

5. Paolini S, Ciappetta P, Domenicucci M, et al. Intramedullary neurenteric cyst with a false mural nodule: case report. Neurosurgery. 2003;52(1):243-246.

6. Menéndez RH, D'Osvaldo DH, Vilariño A, et al. Neurenteric cyst of the ventral craniocervical junction: case report and review of the literature. World Neurosurg. 2019;125:257-260.

7. Shakudo M, Inoue $Y$, Ohata K, et al. Neurenteric cyst with alteration of signal intensity on follow-up MR images. AJNR Am J Neuroradiol. 2001;22(3):496-498.

8. Jung H-S, Park S-M, Kim G-U, et al. Unique imaging features of spinal neurenteric cyst. Clin Orthop Surg. 2015;7(4):515-518.

9. Shukla M, Behari S, Guruprasad B, et al. Spinal neurenteric cysts: Associated developmental anomalies and rationale of surgical approaches. Acta Neurochir (Wien). 2015;157(9):1601-1610.

10. Chen J, Lai $R$, Li Z, et al. Case report series and review of rare intradural extramedullary neoplasms-bronchiogenic cysts. Medicine (Baltimore). 2015;94(49):e2039.

11. Kida K, Tani T, Kawazoe T, et al. A recurrent cervical neurenteric cyst treated anteriorly: safe, gross-total excision facilitated by prophylactic unilateral vertebral artery exposure, microdissection, and spinal cord monitoring-a case report and technical note. Case Rep Orthop. 2018;2018:7620182.

12. Yang T, Wu L, Fang J, et al. Clinical presentation and surgical outcomes of intramedullary neurenteric cysts. J Neurosurg Spine. 2015;23(1):99-110.

\section{Disclosures}

The authors report no conflict of interest concerning the materials or methods used in this study or the findings specified in this paper.

\section{Author Contributions}

Conception and design: all authors. Acquisition of data: Salehani. Analysis and interpretation of data: Howell, Harmon. Drafting the article: Salehani, Howell. Critically revising the article: Salehani, Harmon. Reviewed submitted version of manuscript: all authors. Approved the final version of the manuscript on behalf of all authors: Salehani. Administrative/technical/material support: Harmon. Study supervision: Harmon.

\section{Correspondence}

Arsalaan Salehani: University of Alabama at Birmingham Hospital, Birmingham, AL. asalehani@uabmc.edu. 